\title{
EMDR: Heilsamer Seitenwechsel
}

Traumatherapie mit BILATERALER STIMULATION Andreas Zimmermann

\section{KURZ GEFASST}

1 Die EMDR-Therapie ruft belastende Gefühle und Erinnerungen wach und modifiziert diese unter taktiler, visueller oder auditiver, bilateraler Stimulation.

2 Patientenressourcen und -erkenntnisse stehen dabei im Mittelpunkt, sowie die drei Wirkprinzipien Entkonditionierung, Aufmerksamkeitsteilung und Veränderung des synaptischen Potenzials.

3 Im beschriebenen Fall fand die zuvor hilflose und überforderte Patientin unter der Therapie zu entscheidenden Einsichten über ihre Möglichkeiten, Ressourcen und anstehenden Lebensentscheidungen.

Die Psychotherapeutin und Begründerin der EMDR-Methode, Dr. Francine Shapiro, bemerkte bei einem Spaziergang im Park ein interessantes Phänomen: Einige Ängste und stark belastende Gedanken, die sich aufgrund einer bei ihr diagnostizierten Krebserkrankung eingestellt hatten, verschwanden ohne erkennbare Ursache und traten auch nicht wieder auf. Dies inspirierte sie, nachzuforschen: Was war an diesem Spaziergang anders? Sie erkannte, dass es die Bewegungen ihrer Augen waren, zu denen sie durch die speziellen Lichtverhältnisse verleitet war. Das warf die Frage auf: Konnten diese Reaktionen ursächlich für die Stimmungsverbesserung und das Schwinden der negativen Gedanken sein? Daraus entwickelte Shapiro das Konzept gezielter Augenbewegungen. Sie erprobte es zunächst an Freunden, Bekannten und Kollegen. Dieses Vorgehen nannte sie „bilaterale Stimulation“, eine Links-rechts-Bewegung der Augen.

Sie schloss intensive Studien mit PTBS-Patienten (Posttraumatische Belastungsstörung) an, insbesondere Kriegsveteranen und Missbrauchsopfern. Seit dieser Zeit erfolgen kontinuierlich weitere Schritte, um EMDR (für: Eye Movement Desensitization and Reprocessing) zu evaluieren und die Wirkungsweise dieser Methode wissenschaftlich zu untermauern. Mittlerweile weisen die Studien die hohe Effektivität und nachhaltige Wirkung von EMDR auch über längere Zeiträume nach [1].

\section{Erinnerungen, Gefühle und Verhaltensweisen durch bilaterale Stimulation modifizieren}

Im Rahmen einer EMDR-Therapie soll der Patient unter Anleitung des Therapeuten Erinnerungen an ein Trauma wachrufen. Dabei folgt er in der Regel mit den Augen den Fingern des Therapeuten, der diese schnell und rhythmisch nach links und rechts bewegt. Durch diese bilaterale Stimulation der linken und rechten Gehirnhälfte lassen sich negative Erinnerungen neutralisieren und belastende Gefühle und Verhaltensweisen modifizieren. Die Fingerbeziehungsweise Winkbewegungen finden in einer Geschwindigkeit statt, die vom Patienten als entspannend und zugleich kraftvoll empfunden wird.

\section{Taktil, visuell, auditiv: Welche Reize sind angenehm, welche nicht?}

Besonders häufig und gut bewährt in Therapien ist die bilaterale Stimulation über Augenbewegungen. In den letzten Jahren bieten Therapeuten weitere Reizmöglichkeiten an, darunter taktil (zum Beispiel sanft klopfende Berührungen durch den Therapeuten), auditiv oder visuell (Lichtreize).

Die sorgfältige Absprache (Feintuning) mit dem Patienten für das Vorgehen und die Art der bilateralen Stimulation ist ein wichtiger Therapieschritt. So können als visueller Reiz Eyemotion Glasses eingesetzt werden. Hierbei handelt es sich um eine Lichtbrille, die bilaterale Lichtimpulse in der gewählten Geschwindigkeit auf die Netzhaut sendet. Patient und Therapeut können somit während des Arbeitens nach Bedarf auch Augenkontakt halten. Für manche Menschen ist das Erleben dieser passiven Stimulationsform angenehmer, als den Winkbewegungen zu folgen.

Merke: Der Patient hat immer die Kontrolle über Art, Ort, Tempo, Dauer und Intensität der Reize. 

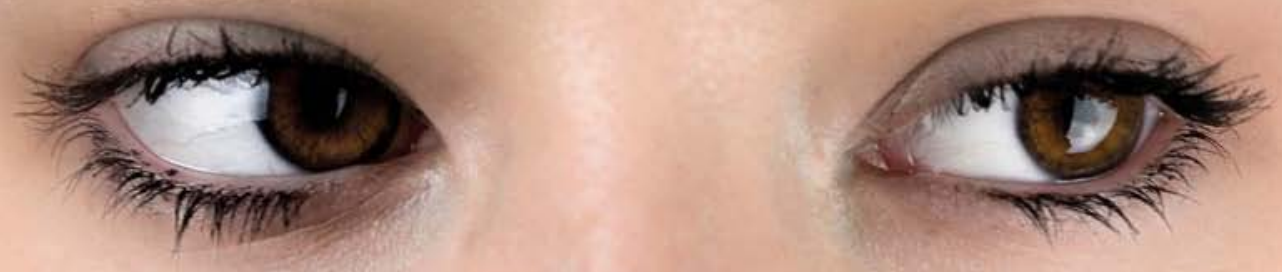

Abb. 1 Bei der visuellen bilateralen Stimulation folgt der Klient in der Regel mit den Augen den Fingern des Therapeuten, der diese schnell und rhythmisch nach links und rechts bewegt (Symbolbild). Quelle: Kirsten Oborny, Thieme Gruppe

\section{Feintuning: Patient bestimmt Ort, Tempo und Intensität der Berührung}

Wenn der Patient sich für die taktile Form der bilateralen Stimulation entscheidet, ist zu klären, ob er vom Therapeuten berührt werden kann oder nicht. Wichtig ist für jede Form von Berührung die Erlaubnis des Patienten. Danach beginnt mit ihm das weitere Feintuning. Zunächst bieten wir leichtes Tapping, ein sanftes Klopfen an unterschiedlichen Körperstellen, an (zum Beispiel Knie, Handrücken, Schulter). An der ausgewählten Stelle wird abgestimmt, wie schnell und intensiv die Berührung sein kann, sodass sie als möglichst kraftvoll erfahren wird. Der Patient hat immer die Kontrolle über Art, Ort, Tempo und Intensität der Berührung.

Für die auditive bilaterale Stimulation eignen sich verschiedene Klangquellen, vom Fingerschnipsen über Klanghölzer bis hin zu Trommeln oder CDs, bei denen spezifische Musikstücke mit bilateraler Stimulation unterlegt sind.

\section{Drei Wirkprinzipien, um Belastendes aus dem Traumaspeicher zu befreien}

Den seelischen Heilungsprozess, der durch EMDR initiiert wird, nannte Francine Shapiro AIP (Acceleratet Information Process), übersetzt: Prozess der beschleunigten Informationsverarbeitung.
Hierbei kommen nach der EMDR-Theorie drei Wirkprinzipien zusammen: Entkonditionierung, Aufmerksamkeitsteilung und die Veränderung des synaptischen Potenzials.

\section{Entkonditionierung: Entspannung nach heftigem Einstieg}

Der Patient wird zu Beginn der Stimulationsphase in der Regel gebeten, mit dem Bild des schlimmsten Momentes seiner Belastung zu starten. Durch die Arbeit mit den Augenbewegungen wird er daraufhin wieder ruhiger und entspannter. Ein parasympathischer Effekt tritt ein.

\section{Aufmerksamkeitsteilung: \\ Frühere Belastung mit dem Jetzt koppeln}

Eine besondere Rolle kommt der Aufmerksamkeitsteilung zu. Der Patient wird zu Beginn der Stimulationsphase aufgefordert, mit einem Teil seiner Aufmerksamkeit in seiner Vorstellung bei dem belastenden Erlebnis zu sein und mit dem anderen Teil den Fingerbewegungen des Therapeuten gezielt $\mathrm{zu}$ folgen. Somit findet eine permanente Hier-und-Jetzt-Orientierung statt.

\section{Veränderung des synaptischen Potenzials: Vom Hot System ins Cool System}

Durch die wechselseitige Aktivierung der Amygdala als paariges Organ wird der wechselseitige Impuls der bilateralen Stimulation 


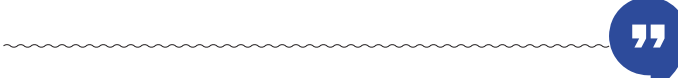 \\ Zurückgebliebene Traumafrag- mente werden durch EMDR zu einer ganzheitlichen Erfahrung.}

an beide Großhirnhälften weitergegeben. Dies regt den ganzheitlichen Verarbeitungsvorgang im Gehirn an. Dabei entsteht ein assoziativer Zugang zu den betroffenen neuronalen Netzwerken.

Die Traumafragmente, die im Hot System (implizites, also unbewusstes Gedächtnis) nach der Traumatisierung zurückgeblieben sind, werden durch EMDR zu einer ganzheitlichen Erfahrung, die im Cool System (explizites, bewusstes Gedächtnis) abgespeichert ist - und somit zu einem Teil der eigenen Lebensgeschichte. Die bilaterale Stimulation fördert auch die Produktion von Acetylcholin. Dieses wird unter anderem in den REM-Phasen unseres Schlafs ausgeschüttet und ist neben seinen vielen anderen Funktionen für die Verarbeitung von Affekten und Assoziationen zuständig.

\section{Zugang zu Ressourcen als Voraussetzung von EMDR}

Ressourcen scheinen in der Psychotherapie das eigentlich wirksame Element der Heilung zu sein. Im Traumaerleben haben sie zur Bewältigung der Situation nicht ausgereicht. So ist es in der The-

\section{HINTERGRUND}

\section{Indikationen und Kontraindikationen der EMDR-Therapie}

EMDR lässt sich insbesondere zur Behandlung der PTBS bei Erwachsenen und Kindern einsetzen. Ferner ist die Methode bei akuten Traumatisierungen indiziert, sofern sichergestellt ist, dass der Patient stabil und für die Behandlung mit EMDR geeignet ist (siehe Kontraindikationen). Wirksam ist EMDR auch bei extremen Trauerreaktionen, Angst- und Panikstörungen, bei der Bewältigung von Unfallfolgen, Phobien, sexuellen Dysfunktionen, chronischen Schmerzen und psychosomatischen Störungen. Ergänzend findet die Therapie auch bei der Bewältigung somatischer Erkrankungen Anwendung. Zudem existieren spezifische Protokolle für die Behandlung von Allergien, Zwängen, Burnout, Tinnitus und Zahnarztangst. Darüber hinaus gibt es Protokolle zur Veränderung unerwünschter Verhaltensweisen bis hin zur Bearbeitung substanzgebundener Süchte.

Klassische Kontraindikationen sind aktuelle psychotische Syndrome, hirnorganische Erkrankungen, reduzierte Ich-Stärke, starker sekundärer Störungsgewinn und fehlende Therapiemotivation. Bei Augenerkrankungen sollte auf eine Stimulation der Augen verzichtet werden. Hier kann jedoch eine taktile oder auditive bilaterale Stimulation eingesetzt werden.

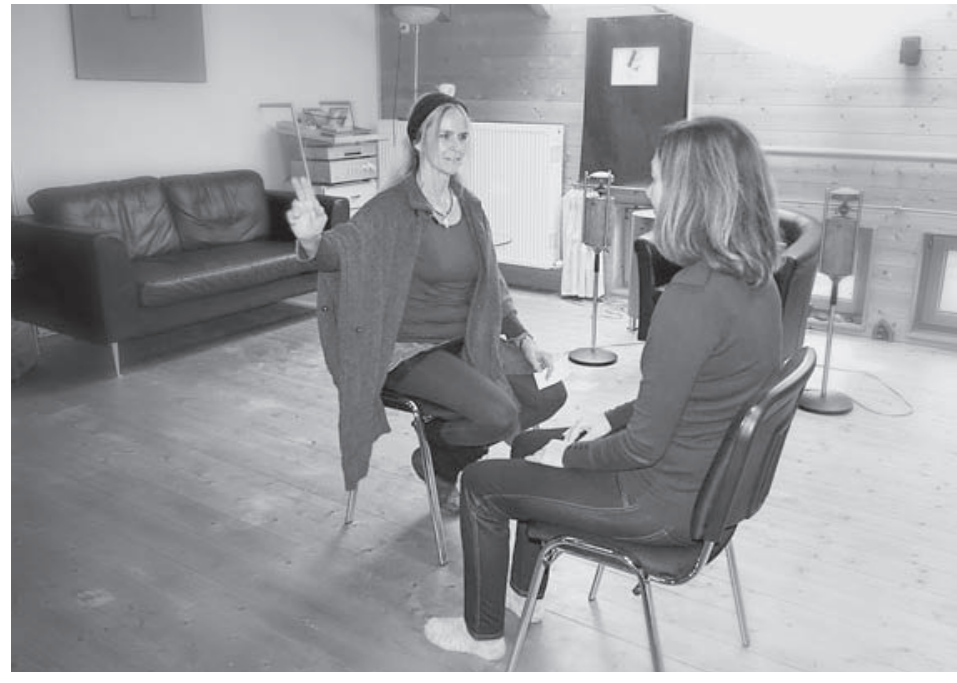

Abb. 2 Setting beim EMDR. Der Therapeut sitzt schräg versetzt zum Klient in einem Abstand, den der Klient bestimmt (Symbolbild). Quelle: Zimmermann A. EMDR für Heilpraktiker. Stuttgart: Haug; 2020

rapie umso wichtiger, Kompetenzen und Ressourcen - auch bereits in der Anamnese - bewusst zu machen und ihre Kraft zu verstärken.

\section{Wer darf EMDR lernen und anwenden?}

EMDR ist eine psychotherapeutische Methode, anwendbar im Rahmen der Sorgfaltspflicht, ob von Ärzten, Psychotherapeuten oder Heilpraktikern. Dies beinhaltet, dass der Therapeut nur Methoden einsetzt, die lege artis erlernt wurden. Das heißt, dass nach einer psychotherapeutischen Grundausbildung die EMDRWeiterbildung selbsterfahrungsorientiert, von Supervisionen begleitet und mit entsprechend notwendigen Ausbildungsinhalten in einem qualifizierten Ausbildungsinstitut absolviert wird. Der Mythos, dass „EMDR“ markenrechtlich geschützt sei, trifft nicht Zu.

\section{Fallbeispiel Überforderung und Hilflosigkeit: Die acht Schritte des EMDR-Protokolls}

Folgender Fall veranschaulicht den Ablauf eines klassischen EMDR-Protokolls: Frau L. (Name von der Redaktion geändert), 33 Jahre alt, in zweiter Ehe verheiratet, zwei Kinder aus erster, ein Kind aus zweiter Ehe, erschien in meiner Praxis zum Erstgespräch. Sie litt seit mehreren Monaten zunehmend an einem starken seelischen Druck, vermehrt auftretender Aggressivität, starkem Gedankenkreisen sowie einem erhöhten, körperlich spürbaren Druck und Brennen in der Herzgegend. Organische Ursachen waren in der Vorgeschichte bereits ausgeschlossen worden. Frau L. erlebte sich selbst in zunehmendem Maß einer Überforderungssituation ausgesetzt, in der sie neben ihrem beruflichen Engagement das gesamte häusliche Umfeld organisieren musste - von der intensiven Begleitung der Kinder (3, 13 und 14 Jahre) bis hin 
zur Versorgung der Mutter, die mit im Familienverbund wohnte. Immer wieder kam es zu Streitereien zwischen ihrem Mann und ihren beiden jugendlichen Söhnen. Auch der Konflikt ihres Mannes mit ihrer Mutter spitzte sich zu. Ihre Reaktion auf diese Probleme war von einem ambivalenten Verhalten geprägt, das sich einerseits durch impulsive und ungewollte verbale, unangemessene Aggression zeigte und andererseits durch zunehmenden inneren Rückzug. Ihre Gedanken kreisten immer mehr um die jeweiligen Konflikt- und Belastungsthemen. Somit fiel es ihr im Lauf der Zeit immer schwerer, klare und konstruktive Gedanken zu fassen und sich zu konzentrieren.

\section{Umfeld, Visionen, Gefüh/smanagement: Welches sind die Ressourcen und Ziele?}

Die Ressourcendiagnostik zeigte ein gutes und kraftvolles soziales Umfeld, repräsentiert durch die Beziehung zu ihren Kindern, der Mutter und ihrem Freundeskreis. Ferner hatte sie eine klare Vision von einer beruflichen Neuorientierung und damit verbundener Selbstständigkeit. Dieses Ziel wurde jedoch nicht von ihrem Mann unterstützt. Als Ressource zeigte sich auch die Verbindung zu ihrem Körper. Ihr Ziel für die gemeinsame therapeutische Arbeit bestand darin, in Situationen angemessen zu reagieren sowie ihre Emotionen besser steuern und kontrollieren zu können. Sie wollte außerdem wieder klar und konzentriert denken und ein freieres, gelösteres Körpergefühl in der Herzgegend empfinden.

\section{Anamnese und Auslösesituation}

In der Anamnese arbeiteten wir Erinnerungs- und Auslösesituationen sowie mögliche Zukunftsängste heraus. Dazu beschäftigten wir uns mit einer Auslösesituation, die unmittelbar vorher stattgefunden hatte: Frau L. war beruflich unterwegs, führte ein Telefonat mit ihrem Mann zu Hause und musste erfahren, dass es wieder Streitigkeiten zwischen ihm und dem 13-jährigen Sohn gegeben hatte. Er war ihrem Sohn offenbar wieder unangemessen und abschätzig begegnet. Während des Telefonats traten unmittelbar wieder das Druckgefühl und das Brennen in der Herzgegend auf. Verstärkt wurde dieses Erleben durch die Unfähigkeit, aus der Ferne regulierend wirksam sein zu können. Auf dieser Grundlage war es ihr auch nicht möglich, klar und authentisch mit ihrem Mann zu kommunizieren. Was sie ihm eigentlich sagen wollte, hielt sie in diesem Telefonat zurück. Diese Situation wählten wir zum Einstieg in die Sitzung aus.

\section{Stoppsignal}

Auch in letzter Instanz vermittle ich den Patienten das Gefühl von Kontrolle. Da manche beim Erleben eines Flashbacks nicht in der Lage sind, zu verbalisieren (vergleichbar einer Blockade des Sprachzentrums), wird ein nonverbales Signal gewählt. Als Stoppsignal (insbesondere bei emotionaler Überforderung) vereinbarten wir das Heben ihrer linken Hand.

\section{Sicherer Ort}

Als sicheren Ort, der in der Anamnese herausgearbeitet wird, beschrieb die Patientin einen kraftvollen Ort in der Natur. Das dor-

\section{HINTERGRUND}

\section{VOC- und SUD-Skala als Gefühlsbarometer}

VOC-Skala steht für Validity of Cognition. Mit ihrer Hilfe bewertet der Patient, wie stimmig eine positive Kognition in Bezug zum schlimmsten Moment des Erlebens auf einer Skala von 1-7 ist. Die Ziffer 1 steht hierbei für maximal unstimmig, 7 für maximal stimmig.

Die SUD-Skala (Subjektiv Units of Disturbance) bemisst auf einer Skala von 0-10, wie belastend der schlimmste Moment für den Patienten ist. 0 steht hierbei für überhaupt nicht, 10 für maximal belastend.

tige Sicherheitsempfinden ging in Resonanz mit einem körperlichen Gefühl von Wärme und Leichtigkeit im Solarplexus, das sich von dort aus in den gesamten Körper ausbreitete.

\section{Auswahl der bilateralen Stimulation}

Die bilaterale Stimulation bildet das Kernstück der EMDR-Therapie und kann taktil, auditiv oder visuell erfolgen. Frau L. entschied sich für das Arbeiten mit Augenbewegungen.

\section{Bewertung}

Der schlimmste Moment in der Auslösesituation war für sie die Unmöglichkeit, aus der Ferne zu intervenieren. Die Vorstellung der Konfliktsituation zwischen ihrem Mann und ihrem Sohn war von dem Körpergefühl des Drucks und Brennens am Herz begleitet. Ihre negative Kognition lautete: „Ich kann nichts tun.“ Ihre positive Kognition formulierte sie folgendermaßen: „Ich kann mich wirksam ausdrücken." Auf der VOC-Skala vergab sie einen Wert von 2 und auf der SUD-Skala eine 8 (Erklärung der Skalen siehe Kasten).

\section{Reprocessing}

Reprocessing stellt die eigentliche EMDR-Prozessarbeit dar. Sie beinhaltet insbesondere die bilaterale Stimulation. Prozessarbeit in der Psychotherapie bedeutet, dass nichts Konkretes geschehen muss oder soll, aber Raum für die individuellen, inneren Prozesse des Patienten vorhanden ist. Wir starteten mit der gewählten Auslösesituation. In den ersten Serien (eine Serie ist eine Abfolge von ca. 40 Sets, ein Set eine Hin-und-Her-Bewegung der Augen) passierte gar nichts. Daraufhin lud ich sie ein, mit dem „nichts“ einfach weiterzugehen. Nach weiteren Serien stellte sich ein leichteres und weiteres Körpergefühl in der Herzgegend ein. Der Druck sowie das Brennen ließen immer mehr nach. Mit diesem ressourcenvollen Erleben gingen wir im Prozess einige Serien weiter, bis Frau L. sich als weiteren Ressourcen der guten Beziehung zu ihren Kindern und ihrer Mutter bewusst wurde. Damit gingen wir im Prozess weiter bis zu einem Zeitpunkt, an dem sie sehr berührt und emotional reagierte und auch Tränen flossen. Mit dem Hinweis „Alles, was auftaucht, darf sein, und auch Tränen dürfen fließen, und Sie gehen weiter mit den Bewegungen meiner Finger und Ihrem inneren Erleben“ folgte sie weiter ihrem Prozess über 
mehrere Serien hinweg, bis plötzlich aus ihr herausbrach: „Ich muss mich von ihm trennen, ich muss ihn verlassen."

Im weiteren Prozess kam es zu mehreren Höhen und Tiefen. Diese waren einerseits von einer klaren Erkenntnis und einer damit verbunden konsequenten Entscheidung, andererseits aber auch von deren Konsequenz gekennzeichnet, darunter schwere, konfliktbeladene Gespräche, emotionale Belastungen für die Kinder und Organisation der Trennung. Nach diesen Prozessinhalten formulierte sie auf einmal: „Ich gehe meinen Weg!“

In den weiteren Serien entwickelten sich nun ein enormes Ressourcenerleben sowie eine hohe Verbindlichkeit ihr selbst und ihrem Lebensweg gegenüber. Es zeigten sich daraufhin positive $\mathrm{Zu}-$ kunftsassoziationen, die ihren Weg kennzeichneten und in ein Abschlussbild mündeten: Sie sah sie sich selbst in einem entspannten friedlichen Ambiente in einem Pavillon sitzen, wo sie in ein Buch schrieb. Nachdem sie den Satz „Ich gehe meinen Weg“ formuliert hatte, fragte ich sie, ob dies eine neue Kognition sei. Sie bejahte und unterstrich, wie kraftvoll diese für sie sei.

\section{Verankerung}

Die Verankerung beschreibt das Ende der Reprocessingphase, das Abfragen der Skalierungen und gegebenenfalls eine Verfestigung der positiven Kognition. Hinsichtlich des VOC-Wertes fragte ich beide Kognitionen ab. Die Erkenntnis „Ich gehe meinen Weg“ bewertete sie mit einer Stimmigkeit von 7, die Kognition „Ich kann mich wirksam ausdrücken“ mit einer 5 (hier lag eine Restunstimmigkeit nach ihrer eigenen Aussage dadurch vor, dass die Trennungsgespräche definitiv nicht einfach und emotionslos ablaufen würden). Den Grad der emotionalen Belastung bewertete die Patientin angesichts des emotional schwierigen, aber notwendigen Schritts der Trennung auf der SUD-Skala mit einer 3. In der Herzgegend beschrieb sie ein gelöstes, freieres und leichteres Gefühl.

\section{Body Scan}

Als Body Scan bezeichnet man das gedankliche Abtasten (scannen) des eigenen Körpers mit dem Ziel, die eigenen Körperempfindungen, Gedanken und Gefühle wahrzunehmen und sich dieser bewusst zu werden, ohne sie zu bewerten. Dabei vergewissert sich der Patient der Leichtigkeit im Körper, in den Gedanken und Gefühlen.

\section{Abschlusstechniken}

Bei den Abschlusstechniken geht es darum, den Patienten stabil aus der Sitzung zu entlassen und ihm für den Zeitraum zwischen den Sitzungen Sicherungs-, Ressourcen- und Überbrückungstechniken an die Hand zu geben. Diese sind zum Beispiel der sichere Ort, das Führen eines Therapietagebuches oder das Malen eines Bildes. Auch das Nutzen eines imaginierten Tresors bietet sich an, in dem inneres Material abgelegt werden kann. Ich lud die Patientin ein, ihr Abschlussbild vom Pavillon zu imaginieren, begleitet von dem Bewusstsein, ihren Weg zu gehen.

In der nächsten Sitzung fragte ich die VOC- und SUD-Werte ab, die sich unverändert stabil gehalten hatten. Wir setzten daraufhin unsere Arbeit am Thema der erlebten Hilflosigkeit fort.

\section{Eignung als Kombinations- oder Einzelthe- rapie, strukturierte oder virtuose Methode}

Das Arbeiten mit EMDR ist ein spannender, dynamisierender Prozess, wie das Fallbeispiel zeigt. Patient und Therapeut können dabei gleichermaßen die Veränderungen wahrnehmen. Die Anwendung beschränkt sich nicht nur auf die Traumatherapie. EMDR verhält sich zu allen anderen Arbeitsmethoden synergetisch. Das Verfahren lässt sich sehr gut mit den anderen Kompetenzen aus dem psychotherapeutischen Werkzeugkoffer verbinden. Dies erlaubt einerseits ein sehr strukturiertes Arbeiten anhand der einzelnen EMDR-Protokolle, die sich jeweils auf spezifische Indikationen ausrichten. Zum anderen ist aber auch freies Arbeiten möglich, in dem sich Intuition und Synergien entfalten können.

Dieser Artikel ist online zu finden:

http://dx.doi.org/10.1055/a-1095-3478

\section{Literatur}

[1] Shapiro F. EMDR - Grundlagen und Praxis. Paderborn: Junfermannsche Verlagsbuchhandlung; 2012

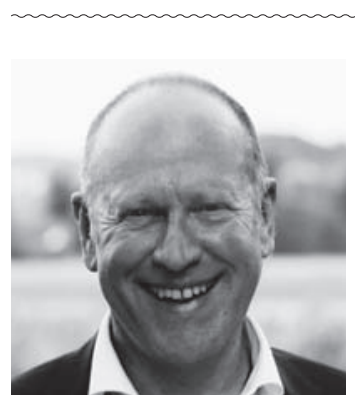

\section{ANDREAS ZIMMERMANN}

Nach mehrjähriger Berufspraxis im Management berät Andreas Zimmermann seit vielen Jahren als Trainer und Coach Unternehmen im Bereich Führung und Change Management. Er leitet in Niederbayern gemeinsam mit seiner Frau eine Akademie mit integrierter psychotherapeutischer Praxis, die Berater, Coaches und Psychotherapeuten in EMDR und mentalen Techniken ausbildet, und schrieb unter anderen das Buch „EMDR für Heilpraktiker“, das im Juni 2020 im Haug Verlag erscheint.

\section{E-Mail: team@emdr-akademie.de Internet: www.emdr-akademie.de}

